\title{
Change of Ground State Configuration Induced by the Stark Shift of Surface States in Some bcc(001) Surfaces
}

\author{
J. G. Che, ${ }^{*}$ Z.Z. Zhu, ${ }^{\dagger}$ and C. T. Chan \\ Physics Department, Hong Kong University of Science and Technology, \\ Clear Water Bay, Hong Kong
}

(Received 4 January 1999)

\begin{abstract}
Using density functional calculations, we found that the ground state configuration of some bcc $(001)$ surfaces are field dependent because different configurations experience different Stark shifts of surface states. When the $\mathrm{W}(001)$ surface is positively biased, an external electric field stabilizes a $c(2 \times 2)$ array of surface vacancies, so that the ground state is no longer the well-established "Debe-King" model. Similar behavior is found for $\mathrm{Mo}(001)$, but not for $\mathrm{Nb}(001)$. The field-induced change of ground state provides a natural explanation for the anomalous behavior of $\mathrm{W}(001)$ inside field ion microscopes. [S0031-9007(99)08932-2]
\end{abstract}

PACS numbers: 68.35.Bs, 68.10.Cr, 73.20.At

In experimental techniques such as scanning tunneling microscopy (STM) and field ion microscopy (FIM), and at the electrode/electrolyte interface, the material surface is subjected to fairly strong external electric $(E)$ fields. Until rather recently, the majority of theoretical calculations have focused on surface properties in the absence of $E$ fields, with only a few exceptions [1]. It is frequently assumed that the surface morphology is not much affected by the field, although there is strong evidence that this may not be the case. For example, potential-induced surface morphological changes are well documented in metal/electrolyte interfaces [2]. Fu and Ho [1] showed that an excess surface charge can induce a missing-row reconstruction in $\mathrm{Ag}(110)$. Pulse-laser field evaporation of fcc $\mathrm{Rh}(001)$ plane gives a $p(2 \times 2)$ structure, stable only if an external $E$ field is applied [3]. Understanding the influence of an $E$ field on surface properties is not only important for interpreting some experimental results, but may also open a new avenue for controlling surface properties. This is why there has been a recent surge in the literature that considers the action of the $E$ field, especially its effect on absorbates [4]. In this article, we examine the effect of $E$ field on some bcc(001) surfaces, and we will show that an external field can have fairly dramatic consequences on systems like $\mathrm{W}(001)$.

The application of $E$ fields will inevitably lead to induced charge rearrangements that cannot be handled easily by empirical models. Metallic screening properties are also difficult to model empirically. The local density formalism can take care of these subtle effects selfconsistently. It is thus the method of choice for studying field induced effects.

The results presented here were performed using the local density functional formalism [5] (Ceperly-Alder local exchange and correlation [6]), and norm-conserving pseudopotentials [7]). The wave functions were expanded by a mixed-basis set of plane waves with kinetic energy up to 11.5 Ry and Bloch sums of numerical orbitals [8]. The bcc(001) surface is modeled by the standard slab geometry. The slabs are nine layers thick, repeated in the (001) direction and separated by vacuum regions of $18 \AA$. The $\vec{k}$ points are sampled on an $8 \times 8$ mesh in the $c(2 \times 2)$ surface Brillouin zone (SBZ) [9]. To include the effect of an external $E$ field, two external uniform charge sheets are placed in the vacuum, one at $9 \AA$ above the slab and one at $9 \AA$ under the slab. The electrons in metallic systems will rearrange themselves to give a surface charge that screens out the field. The surface screening charge and the external charge sheets establish an $E$ field in the vacuum whose magnitude is controlled by the surface charge density of the charge sheet. Atomic coordinates for different models (with and without $E$ fields) are fully relaxed.

The $\mathrm{W}(001)$ surface exhibits a famous $c(2 \times 2)$ displacive reconstruction where the surface atoms are displaced laterally along the $\langle 11\rangle$ direction forming zigzag chains (Debe-King model) [10]. This reconstruction can be viewed as a frozen soft surface $\bar{M}_{5}$ phonon mode [11], so we will label the Debe-King model as $c(2 \times 2) M_{5}$.

We first examine the effect of an $E$ field on $c(2 \times 2) M_{5}$. We found that at zero field, the $p(1 \times 1)$ surface is indeed unstable with respect to a $c(2 \times 2)$ reconstruction in which the surface atoms are laterally displaced along the $\langle 11\rangle$ direction by $\delta \approx 0.26 \AA$, accompanied by a contraction of the first interlayer distance $\left(d_{12}\right)$ by approximately $6 \%$. Our results compare well with experimental data and previous calculations [12]. We next impose an $E$ field. At $E=3 \mathrm{~V} / \AA$, $\delta$ becomes $0.24 \AA$ and $d_{12}$ changes by only $0.01 \AA$, respectively. The $E$ field does not induce noticeable "up-down" staggering displacement of the surface atoms. In other words, the $\bar{M}_{1}$ surface phonon mode remains stiff. Thus, the change in atomic structure is small at typical $E$-field strengths operating in the imaging mode inside a FIM.

We next consider a $c(2 \times 2)$ array of surface vacancies, hereby referred to as $c(2 \times 2)$-vacancy [c(2×2)-V]. In Fig. 1, we compare the local density of states of the surface 


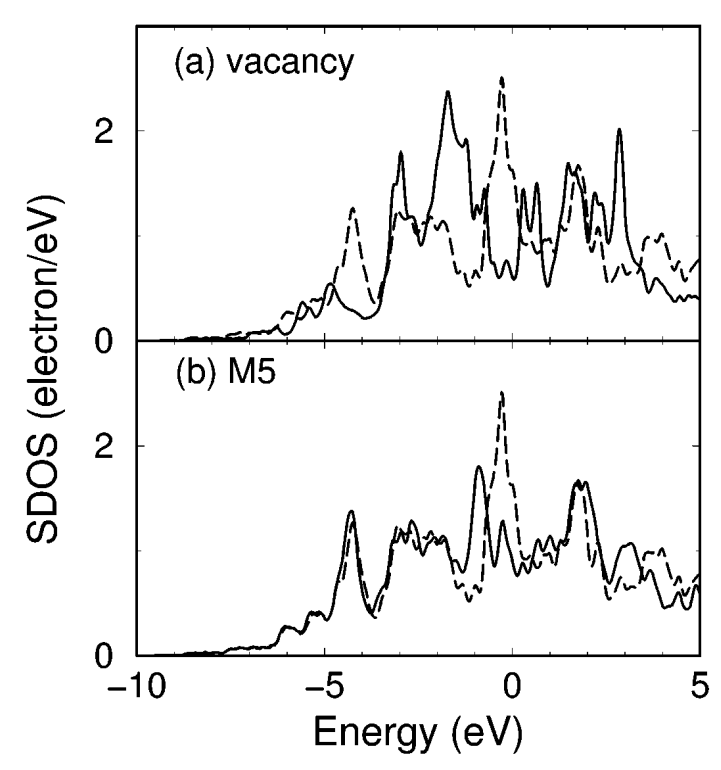

FIG. 1. Surface density of states for W(001) for (a) $c(2 \times 2)$ $V$ and (b) $c(2 \times 2)-M_{5}$; with the SDOS of $p(1 \times 1)$ shown as dotted lines for comparsion.

atoms $(\mathrm{SDOS})$ at zero field for $\mathrm{W}(001)$ in the $p(1 \times 1)$, $c(2 \times 2) M_{5}$ (Debe-King model), and $c(2 \times 2)-V$ structures. Transition metals like Mo and $\mathrm{W}$ have bcc rather than close-packed structures, which is usually attributed to the dip in the bcc DOS near $E_{f}$ for systems with halffilled $d$ shells. When a (001) surface is formed, a peak due to surface states appears near $E_{f}$ in the SDOS of the $p(1 \times 1)$ surface, and this peak is usually associated with the inherent instability of $p(1 \times 1)$. It is well known that the $c(2 \times 2) M_{5}$ reconstruction reduces the SDOS near $E_{f}$ [12], as is evident from Fig. 1(b). What is not well known is that the peak can also be reduced very effectively by the formation of a $c(2 \times 2)$ array of vacancies [Fig. 1(a)]. This correlates well the total energy results, which show that both the $c(2 \times 2) M_{5}$ and the $c(2 \times 2)$ $V$ are lower in energy than the $p(1 \times 1)$ (by 0.07 and $0.09 \mathrm{eV}$ per surface atom, respectively). The local density approximation $(\mathrm{LDA})$ results indicate that $c(2 \times 2)-V$ is marginally $(20 \mathrm{meV} /$ surface atom) lower in energy than $c(2 \times 2) M_{5}$. Even if LDA gets the energy ordering wrong for these nearly degenerate configurations, the field-induced differences to be discussed below should be trustworthy since they are much larger than $0.01 \mathrm{eV}$. In any case, the energetic and kinetic barrier to go from $c(2 \times 2) M_{5}$ to $c(2 \times 2)-V$ is expected to be large, since the transformation requires the removal and transport of half of the surface atoms.

We shall see that $c(2 \times 2)-V$ becomes energetically more favorable if we impose an external $E$ field. We show in Fig. 2 the difference in surface energy between $c(2 \times$ 2)- $V$ and $c(2 \times 2) M_{5}\left(\sigma_{V}-\sigma_{M_{5}}\right)$ as a function of $E$-field strength [13] for the (001) surfaces of $\mathrm{W}, \mathrm{Mo}$, and $\mathrm{Nb}$. In this article, a positive field means that the surface is

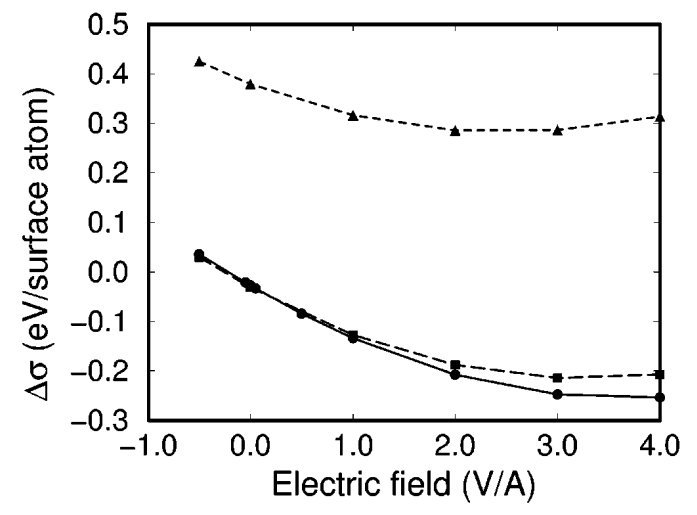

FIG. 2. Differences in surface energies between $c(2 \times 2)-V$ and $c(2 \times 2)-M_{5}$ as a function of $E$-field strength for the (001) surfaces of $\mathrm{W}$ (solid circle), Mo (square), and $\mathrm{Nb}$ (triangle). Negative numbers indicate that $c(2 \times 2)-V$ is more stable. For $\mathrm{Nb}$, the reference energy is $p(1 \times 1)$. The line serves as a guide to the eye.

positively biased, and a negative surface energy difference means that $c(2 \times 2)-V$ is more stable. We will first focus on W(001). We see that when the $E$ field causes depletion of surface electrons, $c(2 \times 2)-V$ becomes strongly favored over $c(2 \times 2) M_{5}$. If we reverse the field, causing extra electrons to go to the surface, $c(2 \times 2) M_{5}$ becomes more stable [14]. We believe that there is already strong experimental evidence for such behavior. It is well documented that $\mathrm{W}(001)$ exhibits a peculiar field-evaporation behavior [15-17]. When $\mathrm{W}(001)$ is field evaporated $[15,16]$ inside a FIM at high temperature, a $c(2 \times 2)$ array of surface vacancies is eventually formed (when observed with an imaging field strength $\approx 4 \mathrm{~V} / \AA$ ) [18]. The original interpretation $[15,16]$ was that the $\mathrm{W}(001)$ surface has "updown" atomic displacement so that the $E$ field selectively evaporates the "up" atoms. It is now well established that the surface atomic displacement is not up-down, but lateral [12]. The unconventional behavior of $\mathrm{W}(001)$ now has a simple interpretation in light of our calculation: $c(2 \times 2)$ $V$ is more stable than $c(2 \times 2) M_{5}$ in the presence of an $E$ field, and the field evaporation helps the system to reach the ground state of a $c(2 \times 2)$ array of surface vacancies. Another indirect evidence is the formation of substitutional surface alloys of many fcc metals with $\mathrm{W}$ and $\mathrm{Mo}(001)$ [19]. These systems can be regarded as fcc elements filling vacancies in the $c(2 \times 2)-V$ configuration. Most of these fcc elements do not alloy with $\mathrm{W}$ in the bulk. Even in a surface environment, electronic structure calculations for the $c(2 \times 2) \mathrm{Cu} / \mathrm{W}(001)$ substitutional surface alloy [20] show that the $\mathrm{Cu}$ atoms have little bonding with the $\mathrm{W}$ atoms. Why should metals like $\mathrm{Cu}, \mathrm{Ag}$, and $\mathrm{Au}$ form $c(2 \times 2)$ surface alloys with $\mathrm{W}(001)$ if they do not like to bond with $\mathrm{W}$ ? The stability of $c(2 \times 2)-V$ must play a role $[19,20]$. We note that these metals are more electronegative than $\mathrm{W}$ on the Pauling scale, and should deplete surface electrons from $\mathrm{W}(001)$. According to our results, 
surface electron depletion stabilizes $c(2 \times 2)-V$, and the fcc elements can then fill in the vacancies to smooth out the surface charge profile. In light of the present result, the formation of these surface alloys may thus arise from "physical" forces rather than direct chemical bonding. We have also considered a $p(2 \times 2)$ array of surface vacancies $[p(2 \times 2)-V]$ for $\mathrm{W}(001)$, and the $E$-field effect is qualitatively similar to that of $c(2 \times 2)-V$, except that it is less dramatic. When $\mathrm{W}(001)$ is positively biased, $p(2 \times 2)-V$ is more favorable than $c(2 \times 2) M_{5}$, but not as favorable in energy as $c(2 \times 2)-V$.

In order to understand the the mechanism underlying such an effect, we show field-induced changes in the SDOS in Fig. 3 for $\mathrm{W}(001)$ in both the $c(2 \times 2)-V$ and $c(2 \times$ 2) $M_{5}$. We see that the field-induced changes in the SDOS for $c(2 \times 2) M_{5}$ are rather small, while the corresponding changes for $c(2 \times 2)-V$ are much more conspicuous, especially near and just above $E_{f}$, where there is a noticeable drop. For a particular configuration, the field-induced atomic displacement is small $(\approx 0.01 \AA$ at $E=3 \mathrm{~V} / \AA)$. Turning on and off the $E$ field for fixed atomic positions accounts for most of the changes seen in Fig. 3. After examining the electronic structure in detail, we found that the change in the SDOS is due to the Stark shift of some surface states (SS) and surface resonances (SR) that have a large amplitude in the vacuum region. The important point to note is that the SS and SR in $c(2 \times 2)-V$ are much more sensitive to $E$ field than those in $c(2 \times 2) M_{5}$.

To illustrate this point, we focus on one $k$ point: $k=$ $(0.5,0)$ in the $c(2 \times 2)$ SBZ. The field-induced changes in the SDOS [21] for this $k$ point are shown in Fig. 4. For both $c(2 \times 2)-V$ and $c(2 \times 2) M_{5}$, the states below $1 \mathrm{eV}$

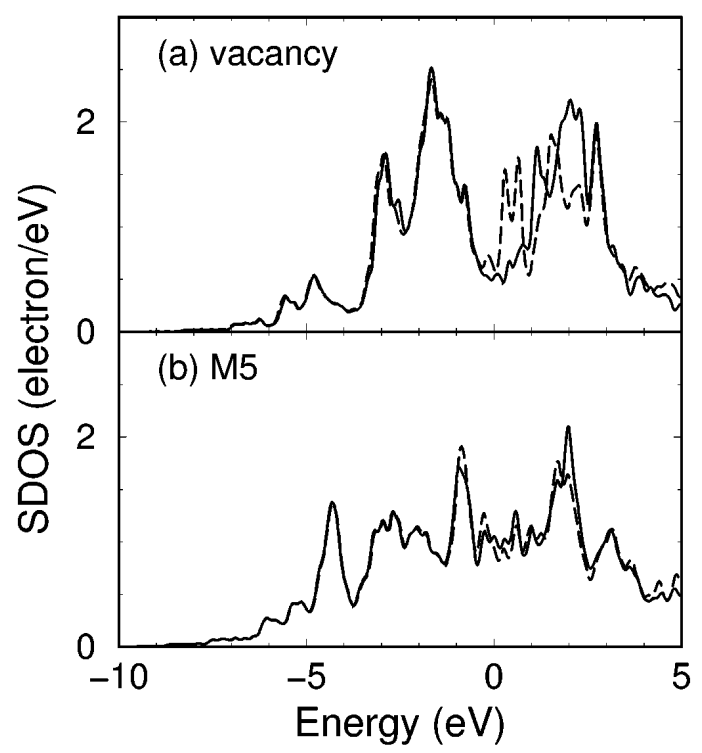

FIG. 3. Surface density of states for $\mathrm{W}(001)$ at $E=3 \mathrm{~V} / \AA$ (solid line) for (a) $c(2 \times 2)-V$ and (b) $c(2 \times 2)-M_{5}$. Dotted lines are zero field SDOS. Note the bigger changes in (a). $E_{f}$ is set at $0 \mathrm{eV}$. show little change. These are bulk states, and they are not much affected since the $E$ field is efficiently screened. For $c(2 \times 2)-V$, states such as those labeled $a$ and $b$ experience significant upward Stark shift. (There are bulk states with energy slightly below $b^{\prime}$, and these bulk states are not affected by the $E$ field.) We found that these are surface states with large lumps of their charge density in the vacuum region. They have large projections on $d_{3 z^{2}-r^{2}}$, and are thus sensitive to an $E$ field normal to the surface. In $c(2 \times 2) M_{5}$, the surface states are in general less sensitive. For example, the surface state (c) is hardly moved by an $E$ field. Its charge density is "in plane" and has predominately $d_{x^{2}-y^{2}}$ and $d_{x y}$ character. The unoccupied surface state labeled (d) has $d_{x z+y z}$ character, making it more sensitive to the $E$ field, but it is still less sensitive than the surface states or resonances in $c(2 \times 2)-V$.

As the $E$ field cannot penetrate into the bulk, it is the surface states/resonances that matter. We see that the surface states and resonances in $c(2 \times 2)-V$ and $c(2 \times$ 2) $M_{5}$ have rather different characters, leading to different behaviors in an external $E$ field. Since this effect is electronic in origin, we expect similar behavior for Mo, which is isoelectronic with $\mathrm{W}$. On the other hand, other bcc systems like $\mathrm{Nb}$ may behave differently. Extending our calculations to $\mathrm{Mo}(001)$ and $\mathrm{Nb}(001)$, we found that this is indeed the case. We reexamine Fig. 2, which also shows the surface energy differences between $c(2 \times 2)$ - $V$ and $c(2 \times 2) M_{5}$ as a function of external field for Mo and $\mathrm{Nb}(001)$. For $\mathrm{Mo}(001)$ [22], we found that (i) $c(2 \times 2)-V$ is competitive with $c(2 \times 2) M_{5}$ at zero field, (ii) positive bias of the surface favors $c(2 \times 2)-V$ and negative bias

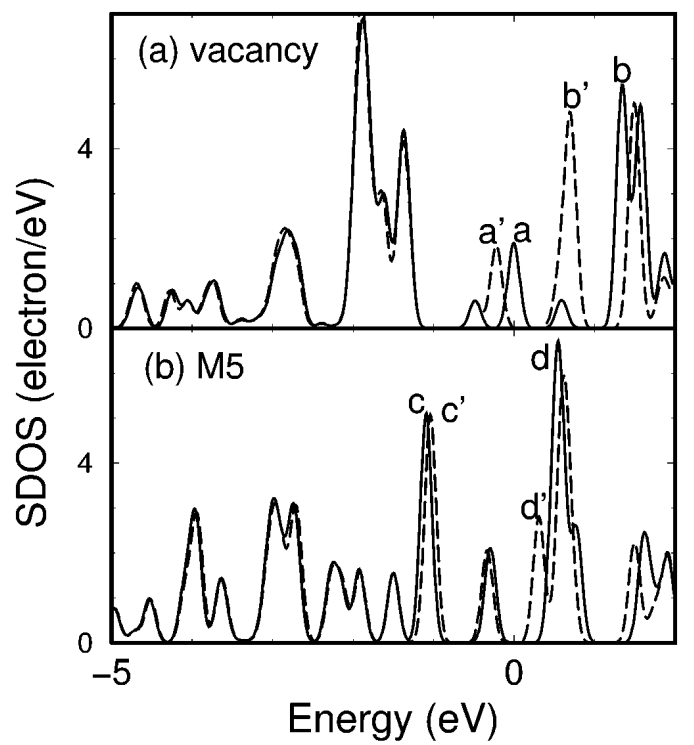

FIG. 4. SDOS at $k=(0.5,0)$ of the $c(2 \times 2)$ SBZ (a) for $c(2 \times 2)-V$, (b) for $c(2 \times 2)-M_{5}$. Dotted and solid lines are for $E=0$ and $E=3 \mathrm{~V} / \AA$, respectively. Symbols $a, b, c$, and $d\left(a^{\prime}, b^{\prime}, c^{\prime}\right.$, and $\left.d^{\prime}\right)$ label surface states when the $E$ field is on (off). 
favors $c(2 \times 2) M_{5}$; (iii) it is the significant Stark shift of surface states/resonances near $E_{f}$ that favors $c(2 \times 2)$ $V$ over $c(2 \times 2) M_{5}$. We now consider $\mathrm{Nb}(001)$, which behaves differently. We note that (i) $p(1 \times 1)$ is stable: there is no $c(2 \times 2) M_{5}$ reconstruction; (ii) $c(2 \times 2)-V$ is much higher than $p(1 \times 1)$ : we do not expect surface vacancy arrays to be formed easily; (iii) imposition of $E$ field does not change the picture: up to a bias of $4 \mathrm{~V} / \AA$, $p(1 \times 1)$ remains the ground state. We found that the surface states and resonances responsible for the field response of Mo and W surfaces are actually there, and they experience similar Stark shifts under $E$ fields. However, $\mathrm{Nb}$ has fewer electrons and thus the SDOS of this group of surface states peaks at an energy higher above $E_{f}$, therefore the effect is small, far from sufficient to tilt the balance as $c(2 \times 2)-V$ is too high in energy. $\mathrm{Nb}$ thus behaves differently.

In short, an external $E$ field can have rather interesting effects on surface morphology. The field induced changes are by no means a priori obvious until detailed calculations reveal the electronic mechanisms at work at the atomic level. It is particularly interesting and surprising that such effects should appear in bcc metals like W and Mo, where the bulk DOS is rather symmetric about $E_{f}$. However, the spatial distribution of the surface states and resonances near $E_{f}$ can a have strong dependence on surface morphology, making the rougher $c(2 \times 2)$ - $V$ configuration much more sensitive to external fields. This may be the first example that shows that the Stark shift of surface states can actually change the surface ground state configuration. Recognizing such an effect helps us understand some intriguing properties of $\mathrm{W}(001)$, such as its peculiar behavior inside FIM and the formation of $c(2 \times 2)$ surface alloys without conspicuous chemical bonding.

C. T. C. thanks Dr. H. Haas and Dr. K. M. Ho for helpful communications, and Dr. B. Meyer, Dr. C. Elsaesser, and Dr. M. Fahnle for making available their code, and Dr. M. Yamamoto for sharing computational resources. This work is supported by RGC Hong Kong through HKUST6128/98P.

*Permanent address: Department of Physics, Fudan University, Shanghai, China.

†Permanent address: Department of Physics, Xiamen University, Xiamen, China.

[1] See, e.g., C. L. Fu and K. M. Ho, Phys. Rev. Lett. 63, 1617 (1989); G. C. Aers and J. E. Ingesfield, Surf. Sci. 217, 367 (1989); K. Watanabe and T. Satoh, Surf. Sci. 287-288,
502 (1993); J. Neugebauer and M. Scheffler, Surf. Sci. 287-288, 572 (1993).

[2] See, e.g., J. Wang et al., Science 255, 1416 (1992); D. M. Kolb, Prog. Surf. Sci. 51, 109 (1996), and references therein.

[3] C. F. Ai and T. T. Tsong, Surf. Sci. Lett. 43, 1521 (1997).

[4] See, e.g., H. Ness and A. J. Fisher, Phys. Rev. B 55, 10081 (1997); A. Pomyalov, Phys. Rev. B 57, 8989 (1998); S. Clarke et al., J. Phys. Condens. Matter 10, 7777 (1998); H. C. Akpati et al., Surf. Sci. 401, 47 (1998); K. P. Bohnen and D. M. Kolb, Surf. Sci. 407, L629 (1998).

[5] See, e.g., Theory of the Inhomogeneous Electron Gas, edited by N.H. March and S. Lundqvist (Plenum, New York, 1983), and references therein.

[6] D. M. Ceperley and B. J. Alder, Phys. Rev. Lett. 45, 566 (1980); J. P. Perdew and A. Zunger, Phys. Rev. B 23, 5048 (1981).

[7] G. B. Bachelet and M. Schlüter, Phys. Rev. B 25, 2103 (1982).

[8] C. Elsasser et al., J. Phys. Condens. Matter 2, 4371 (1990); K. M. Ho et al., J. Phys. Condens. Matter 4, 5189 (1992).

[9] When checked with a $12 \times 12$ grid, the change in surface energy is less than $1 \mathrm{mRy}$ per surface atom.

[10] M. K. Debe and D. A. King, Phys. Rev. Lett. 39, 708 (1977); T. E. Felter, R. A. Barker, and P. J. Estrup, Phys. Rev. Lett. 38, 1158 (1977).

[11] See, e.g., A. Fasolino, G. Santoro, and E. Tosatti, Phys. Rev. Lett. 44, 684 (1980).

[12] M. S. Altman, P. J. Estrup, and I. K. Robinson, Phys. Rev. B 38, 5211 (1988); C. L. Fu and A. J. Freeman, Phys. Rev. B 37, 2685 (1988); R. Yu, H. Krakauer, and D. Singh, Phys. Rev. B 45, 8671 (1992).

[13] The difference in surface energy between two configurations has well-defined values in our calculation since they vacuum field energy substracts out.

[14] We cannot impose a very strong field in the latter case because electrons will escape to the vacuum.

[15] A. J. Melmed et al., Phys. Rev. Lett. 43, 1521 (1979).

[16] R. T. Tung, W. R. Graham, and A. J. Melmed, Surf. Sci. 115, 576 (1982).

[17] T. T. Tsong and J. Sweeney, Solid State Commun. 30, 767 (1979).

[18] See, in particular, Fig. 7 of Ref. [16].

[19] G. A. Attard and D. A. King, Surf. Sci. 188, 589 (1987); G. A. Attard and D. A. King, Surf. Sci. 222, 360 (1989); E. Bauer et al., J. Appl. Phys. 45, 5164 (1974).

[20] D. Singh and H. Krakauer, Surf. Sci. 216, 303 (1989).

[21] The SDOS has been broadened by a Gaussian of width $0.1 \mathrm{eV}$.

[22] Experimentally observed reconstruction of $\mathrm{Mo}(001)$ is actually $c(7 \sqrt{2} \times \sqrt{2})$ [see, e.g., R. S. Daley et al., Phys. Rev. Lett. 70, 1295 (1993)], but the $c(2 \times 2) M_{5}$ model should be a good approximation for the consideration of field response. 